\title{
Modeling techniques for decentralized energy systems applied in smart grids
}

\author{
Nasima El assri ${ }^{1 *}$, Samira Chabaa ${ }^{2}$, Khadija Lmesri $^{1}$, Mohammed Ali Jallal ${ }^{1}$ and Abdelouhab Zeroual ${ }^{1}$ \\ ${ }^{1}$ I2SP Team, Physics Department, Faculty of Sciences Semlalia, Cadi Ayyad University, Marrakesh, Morocco \\ ${ }^{2}$ Industrial Engineering Department, National School of Applied Sciences, Ibn Zohr University, Agadir, Morocco
}

\begin{abstract}
Historically, power grids have emerged as the most economical way to match diversified generation resources. After the Second World War, all advanced countries chose to develop a centralized electrical system to transport energy throughout the national territory and then distribute it to the various points of consumption. In this centralized management, production is adapted to an ever-increasing demand, driven by economic and demographic developments. Gradually, the consumption profile has changed: the development of electrical uses (particularly heating and air conditioning), leading to consumption peaks that are increasingly difficult to satisfy. The appearance of the electric vehicle reinforces these growing imbalances between supply and demand. Thus, the production profile has gradually changed: production has moved closer to the places of consumption but has become more variable, such as wind and solar energy (known as intermittent sources of energy production). In this transforming energy view, these historical centralized and unidirectional networks reach saturation and need to be modernized by turning to a decentralized model. In this perspective, the development of smart grids (SG) is taking place. This paper aims to define decentralized energy systems that provide various benefits and cause significant challenges. Finally, we propose several techniques that are highly efficient in modeling and controlling smart grid systems in order to help decision-makers to address complex problems.
\end{abstract}

Keywords. Energy systems, centralized, decentralized, Smart-grid, complex system, modeling techniques

\section{Introduction}

With the urging ecological, technical, and social problems of energy. Smart grid is one of the most influential and practical solution to this issue. By gradually changing the existing power grid system into decentralized energy systems that are different in size, elements, and strategies [1].

Contrary to centralized energy supply, decentralized energy supply is the provision of energy through smaller plants closer to the consumers. This definition has a geographic as well as a quantitative component. The decentralized energy conversion plants are located where energy is needed, and the energy is provided by relatively more numerous but much smaller plants compared to the central energy supply. This results in new requirements for energy management, grid operation, and protection technology. Decentralized power systems offer a wealth of environmental, economic, technical, and social advantages for consumers: lower carbon power with greater flexibility.

There are several options: the continuation of the current centralized system, a system where electricity production is totally decentralized, and lastly a hybrid system where a centralized network and local loops of various sizes would coexist. These systems have substantial impacts on smart grids. These systems, known as complex, include subsystems whose actors have divergent objectives whose overall behavior cannot be deduced of these components. In order to help decisionmakers to resolve complex problems, modeling techniques is an essential step. In this paper, we present the advantages and disadvantages of these decentralized energy systems, and we propose modeling techniques that help in designing reliable and efficient smart grids.

Abbreviations

CHP Combined heat and power

DES Decentralized energy system

PV photovoltaic

RES Renewable energy sources

SG Smart grid

*Corresponding author: nasima.elassri@email.org 


\section{Centralized I Decentralized energy system}

A decentralized energy system is characterized by locating energy production facilities closer to the site of energy consumption. A decentralized energy system allows for more optimal use of renewable energy sources (RES) and combined heat and power (CHP), reduces fossil fuel use, and increases eco-efficiency. A DES is a relatively new approach in the power industry in most countries. Traditionally, the power industry has focused on developing large, central power stations and transmitting generation loads across long transmission and distribution lines to consumers in the region. Decentralized energy systems seek to put power sources closer to the end-user. End users are spread across a region, so sourcing energy generation in a similar decentralized manner can reduce the transmission and distribution inefficiencies and related economic and environmental costs [2].

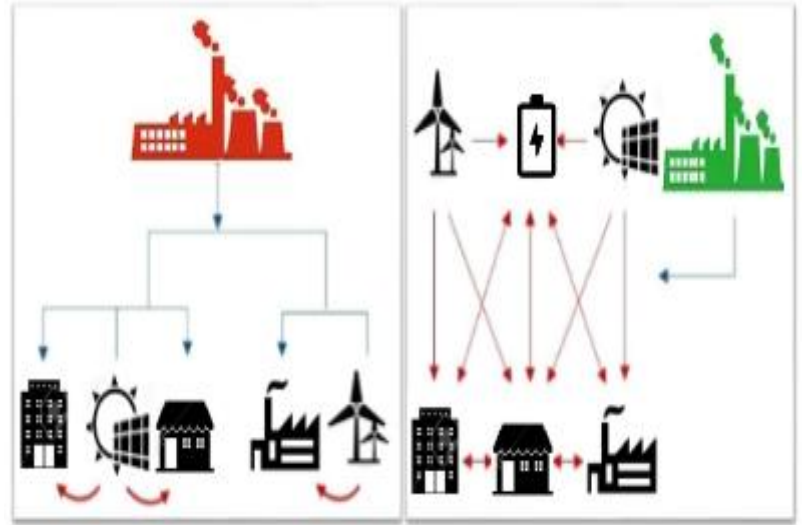

Fig. 1. Schematic representation of a centralized (left) versus decentralized (right) energy system

The evolution of the power system will impact the entire energy chain, from generators to transmission and distribution to individual consumers (table 1).

Table 1. Characteristics of traditional system (left) versus the DES (right).

\begin{tabular}{|c|c|c|}
\hline & Today's electricity & Tomorrow's choices \\
\hline Production & Few large power plants & Many small power producers \\
\hline \multicolumn{3}{|c|}{ y national } \\
\hline \multicolumn{3}{|l|}{ Transmission } \\
\hline \multicolumn{3}{|l|}{ Distribution } \\
\hline Consumer & Passive, only paying & Active, participating in the system \\
\hline
\end{tabular}




\section{Strengths in using a decentralised energy system}

While our current system helped our country grow and develop, there is now an opportunity to grow in an exciting new direction. There are several benefits to moving toward a decentralized power grid, and solar power can be a significant component for achieving this transition. Let us look at the benefits [3]:

\section{Environmental}

- The integration of decentralized CHP into the electricity market increases the global heat and power system's efficiency and thereby reduces harmful greenhouse gas emissions [4].

\section{Economic}

- Distributed generation sources often have lower capital costs per project compared to large central power plants.

- In some circumstances, off-grid distributed generation can reduce the need for expensive transmission and distribution network expansion.

- Lower losses through the lengthy transmission of electricity increase eco-efficiency. Reducing losses in transmission and distribution and the incremental addition to capacity through distributed generation can help defer investment in large central power plants.

- Decentralized siting of energy generation facilities requires decentralized businesses to construct, operate and maintain the facilities, creating opportunities for local business and job creation.

\section{Technical}

- Distributed generation projects provide flexibility due to power-to-heat technologies, storage capacities, and their small size and short construction lead times compared to larger central power plants [5].

- A decentralized energy system influence in managing optimal energy efficiency [6]. Increased information about energy flows from smart meters can make consumers more conscious of their use. Through on-site energy production, consumers of energy become producers and have a more significant economic stake inefficient production and consumption.

- Fewer Upgrades: Between maintenance and capacity upgrades, there is an ongoing need to invest in our existing power grid. However, a de-centralized system spreads and defrays those costs significantly [7].

\section{Social}

- Without a grid connection, communities typically use expensive and unsustainable fossil fuels to generate electricity. Decentralized energy systems can bring stability to off-grid electrification and decrease fossil fuel consumption. By exploiting local green energy resources such as solar and wind power, DESs in rural areas can provide access to energy and facilitate economic growth.

\section{Challenges in using decentralised energy system}

The study concludes that for every advantage that decentralization offers, there is a disadvantage [8]. For example:

- Energy decentralization needs investment: energy production infrastructures have to be built, connected to the network, and need to invest in energy storage infrastructure, especially in the case of renewable energies such as wind and solar, which do not produce continuously.

- Distributed generation sources often have higher capital cost per $\mathrm{kW}$ due to the interconnection transaction costs.

- The high capital costs and long life cycle of existing transmission and distribution infrastructure make it difficult to upgrade to more efficient infrastructure.

Things are progressing relatively slowly, but in the future, the energy transition will necessarily involve decentralization. More ecological, more manageable, less dangerous, and less expensive in the long run, decentralized energy should be at the heart of the energy transition from now on.

\section{Options}

Countries have several options: maintaining the current centralized system, implementing a fully decentralized model, and finally, a hybrid system where a centralized network and local loops of various sizes would coexist.

The first option is the safest in the short term, but it may prove untenable in the long term if economic agents turn to seemingly cheaper self-generation technologies. The second option is costly, even unrealistic in the short term, but it is probably viable in the long term if electricity storage develops at a reasonable cost and if agents accept to regulate their consumption according to the rarity of renewable energy supply and the price signal. The third option is certainly the most comfortable, but it can also be very costly, as it relies on a dual system and therefore on a structural oversupply for which investments will have to be paid.

In any case, the public authorities will have to invest, encourage innovation, arbitrate on technical standards and control the sector.

\subsection{Centralized energy system}

This first option corresponds to the current situation of the electricity system, which guarantees continuity of service for all and equal treatment of consumers through tariff equalization. In the absence of technologically mature decentralized storage solutions, transmission and distribution networks remain essential if we want to absorb and distribute the energy produced by intermittent and uncontrollable RE (wind, tidal, solar). 
To integrate the growing share of these renewable energies into the power system, it is necessary to define their rate of development and their optimal location.

\subsection{Totally decentralized energy system}

This second option is based on generalized local control of energy choices, with quasi-autonomous systems governed by cities, neighborhoods, or citizens' associations via local loops. This model implies the closure of most centralized installations and massive investments in small-scale renewable production means, relying on significant serial and network economies at the local level. It leads to a new solidarity between metropolises and the surrounding territory, which will become their energy producer.

In this scheme, the only function of the national grid is to link the local loops together to pool certain flexibility services, such as intelligent storage management or dynamic supply and demand management.

As production variations are continuous and sometimes unpredictable, consumers must be able to adapt their consumption. They need to be equipped with connected devices controlled by intelligent software according to signals, particularly price signals.

This scenario is only really possible if inter-seasonal electricity storage is developed on a massive scale and at low cost. Indeed, consumption peaks are in winter, while solar production takes place in summer and electrochemical battery technology does not allow for several months of storage (see graph below).
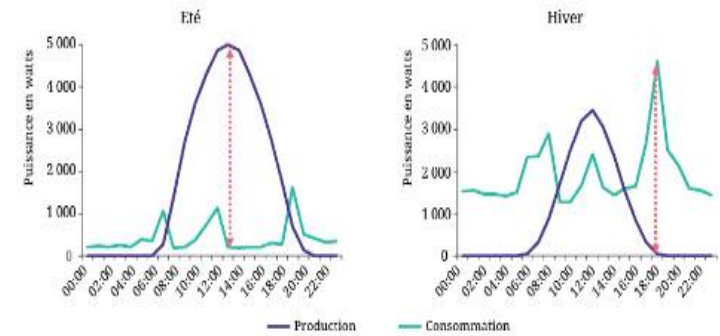

Fig. 2. Daily consumption and production of an individual with a PV panel

Eventually, if all the technologies become available at a reasonable cost, this model may prove to be efficient and correspond to social demand. In this option, the government plays a role as referee and regulator of the activity of microgrid managers, by setting and enforcing common technical operating standards.

\subsection{An intermediate system}

This mixed model combines decentralization and grid assurance, allowing citizens to come together in microgrids of various sizes connected to the centralized grid. In these areas, consumers have a permanent interest in making choices between local supply and the centralized grid, which they use in the event of insufficient or excess local production. They can choose the quality of their power by equipping themselves with storage technologies or by contracting with the grid, which plays an insurance role for them. Production exchanges can take place between microgrids, but the physical transfers are technically managed by the centralized grid, which must maintain a good overall view of the system and ensure the general balance between supply and demand.

In this third option, the investments are carried by both the local systems and the centralized system.

This model has the advantage of better responding to social demand while continuing to ensure security of supply. On the other hand, it has the disadvantage of doubling the traditional network and local loops, and therefore requires very heavy investments that users may not be willing to pay for.

\section{Modeling techniques}

Current energy systems include a societal component due to the impact of users. These complex systems contain numerous sub-systems which actors have conflicting even antagonist objectives.

Considered as the evolution of the current Power Grid, the Smart Grid is a perfect example of complex system. This term refers to an electrical optimized network integrating the behavior and actions of users (generators, consumers, consumactors, etc.). These Power Grid's upgrades aim to improve quality and security, to reduce environmental impact. But current simulations are done on a specific technology and with a limited evolution.

Challenges of Smart Grid are related firstly to the heterogeneity of actors with different interests and secondly to a spatial and temporal multiscale complexity. Smart Grid actors are in a competitive access to a shared resource. For example, consumers express energy requirements with minimal cost while producers have to manage consumption, peaks and to maximize their profits. Dynamics behaviors and relations between entities are difficult to model. External and internal pressures as energy pricing, energy losses or the erratic production of renewable energies influence on the structure, the organization and the behavior of the subsystems and the overall system. Moreover, a bad management causes various structural problems like brownout, blackout, or partial failures.

We propose modeling techniques to solve the Smart Grid. We also suggest some theories to enhance the structural model, to integrate dynamic pricing in order to make the model as efficient as possible.

\subsection{Cartesian analysis and systemic analysis}

In order to autonomously control the Smart Grid, it will be necessary to optimally manage transmission, 
distribution, consumption and communication. Specialists already offer many Smart Grid models.

The majority of studies on Smart Grids use a Cartesian analysis method also called top-down. The system is divided into modules: optimization, network structure, communication technologies and security; and into elements or agents: users, energy, controllers and data. The simulations aim to optimize the global objective function, which varies from one model to another.

The classical methods of study, based on a Cartesian analysis, fail to model complex systems. Taking into account the entropy of these systems, it is not possible to model each part separately nor to model the whole system without considering each part independently.

The development of biology and electronics (Von Bertalanffy and G. Wiener at the end of the 1940's) allowed the establishment of the second type of analysis: the systemic or bottom-up analysis. The problem is studied by focusing on the relationships between the elements.

This method does not aim to provide a unique answer to a problem, but a replacement of the problem that can be used as a model for the simulation of its process and behaviors. The systemic analysis is based on the analysis of the details relevant to the action carried out and to the objectives defined.

The vast majority of Smart Grid studies employ a Cartesian method of system analysis. Although the results are satisfactory, these models become memory intensive with large instances, and impose technologies without being able to study their impact on a large scale or in another framework. We are thus confronted with two shortcomings: a local model that is not exhaustive and predefined, and a Cartesian analysis that is not relevant in a larger model [9].

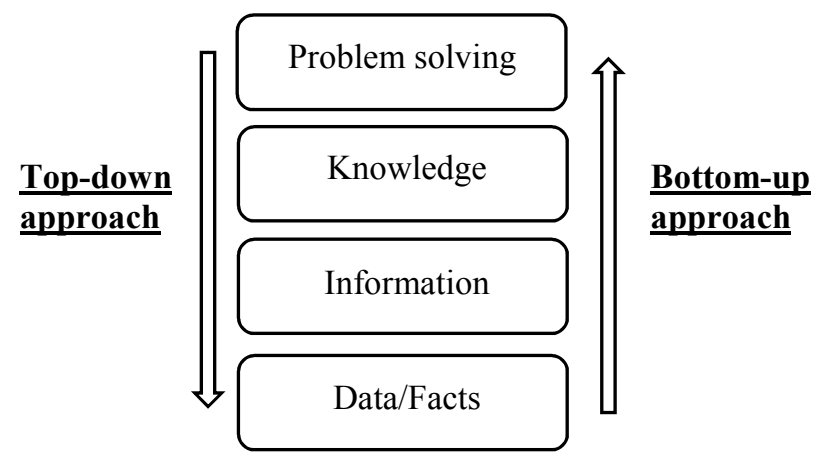

Fig. 3. Top-Down or Bottom-Up Analytics

\subsection{Integrative modeling}

The optimization of a complex system is difficult and requires a specific methodology. Smart Grids aim at smoothing the consumption curve, reducing overall consumption, balancing supply and demand, and integrating new technologies. The constraints are to send orders/energy needs and optimize energy flows, perform preventive maintenance, control permutations and minimize loads to optimize investments, while dealing with renewable energy generation and storage.
The optimization of the subcomponents must meet local criteria, the complexity due to distributed systems and global objectives.

We propose an integrative model to solve the Smart Grid: each component of our model allows to consider one aspect of the grid and it is their intricacy that achieves an efficient modeling. Relations between entities take into account the complex dynamics and an overall supervisor allows network monitoring to detect critical thresholds or to plan consumption schemes [9].

\subsection{Tools for model improvement}

In this section, we will discuss the different theories needed to make the smart grids adaptive, autonomous, and efficient.

\subsubsection{Pretopology theory}

Pretopology is a mathematical theory for modeling the concept of proximity. However, in the context of the integration of renewable energies, it is necessary to express the notions of proximity in a realistic and multidimensional way. If we take the example of wind turbines, we do not talk about metric proximity, but about functional proximity: the weather influences the electrical output produced, so the closest wind turbine in terms of distance is not necessarily the one that will be able to provide us with the required energy [10]. This theory models the behavior of the Smart Grid in a more realistic way. Moreover, the modification of a single criterion only leads to the update of the assigned pretopology, and not to a total restructuring of the grid.

\subsubsection{Percolation theory}

Percolation theory is a branch of statistical physics and mathematics that deals with the characteristics of random environments.

The mathematical model is based on a network of nodes and the connections between each pair of neighbors can be open with probability $\mathrm{p}$, or closed with probability (1-p), and we assume that they are independent.

Electricity networks provide an interesting example of the percolation phenomenon: we consider $\mathrm{n}$ consumers, where each consumer is connected to its neighbors by links of random efficiency $p$. The structure of the electrical distribution network is completely random. There is therefore a proportion (1-p) of links that can be randomly destroyed, which does not result in any visible power outage for the consumers: the power transmission is ensured since there is the possibility to pass through relay entities if $p$ is higher than a critical value Pc called the percolation threshold. Below the Pc, the probability that two entities can communicate directly or indirectly is very low [10].

\subsubsection{Game theory}

Game theory is a field of mathematics that aims to establish and study the mathematical principles and rules 
that can be used to analyze the different types of behavior and possible outcomes in a strategic interaction between several decision-makers.

grids involve various actors with divergent interests: the state, the consumer, and the companies are in perpetual conflict on the economic domain. This is very close to aspects of game theory [10]: it is indeed a strategic problem in which the actions of each actor will influence the other actors.

Game theory guarantees minimal costs for the consumer and the producer. Mathematical methods of flattening the load curve will impact the prognosis in order to limit consumption peaks and thus guarantee a constant low price [11].

\subsubsection{Neural network}

Artificial intelligence is in continuous development and is today achieving considerable success using increasingly complex neural networks. The common point between the two types of electrical networks and neural networks is that they are composed of many interconnected layers.

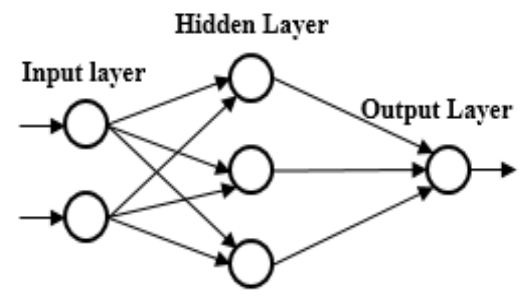

Fig. 4. A simple neural network

the objective is the correlation between Smart Grids (the future smart electrical network) and the science of Machine Learning and especially neural networks in order to predict the consumption [12], guarantee low price [13], and optimize electrical energy chain [14].

\section{Conclusions}

The heterogeneity of its elements, their diverging interests, the diversity of subsystems makes Smart Grids challenging to model by a Cartesian analysis. We have therefore adapted the systemic analysis based on the study of agents and their interaction.

A Smart Grid is composed of sub-systems, with several dynamics and interests. The optimization of a complex system combines various theories. We propose integrative modeling in order to consider each aspect of the Smart Grids modeling problems.

We also present efficient tools needed to make the grid adaptive, autonomous, and efficiently model smart grids to predict the consumption of electrical energy, limit consumption peaks, and guarantee a constant low price.

The Future objective will implement modeling techniques in buildings as the first pillars of SG to ensure optimal systems.

\section{References}

1. M. Ahat, S. Ben Amor, M. Bui, A. Bui, G. Guérard, C. Petermann, Smart Grid and Optimization, in American Journal of Operations Research, Vol.3, $\mathrm{n}^{\circ} .1 \mathrm{~A}, 11 \mathrm{p}$, (2013).

2. « low carbon green growth road map for Asia and the pacific fact sheet | decentralized energy system.pdf», p.1, Available on: https://www.unescap.org/sites/default/files/14.\%20FSDecentralized-energy-system.pdf

3. « low carbon green growth road map for Asia and the pacific fact sheet | decentralized energy system.pdf», p.2-3, Available on: https://www.unescap.org/sites/default/files/14.\%20FSDecentralized-energy-system.pdf

4. W. Schellong, T. Schmidla, I. Stadler, F. Strümpler, Integration of cogeneration systems into smart grids, In International Symposium on Power Electronics Power Electronics, Electrical Drives, Automation and Motion (pp. 1422-1427). IEEE. (2012, June).

5. C. Bergaentzlé, I. G. Jensen, K. Skytte, O. J. Olsen, Electricity grid tariffs as a tool for flexible energy systems: A Danish case study, Energy Policy, 126, 1221, (2019).

6. V. Bakker, A. Molderink, M. G. Bosman, J. L. Hurink, G. J. Smit, on simulating the effect on the energy efficiency of smart grid technologies. In Proceedings of the 2010 Winter Simulation Conference (pp. 393-404), IEEE, (2010, December).

7. «Mountain Power solutions | the benefits of decentralized power production ", Available on: $\mathrm{http}: / /$ mountainpowersolutions.com/the-benefits-ofdecentralized-power-production/

8. «2009 - science for environment policy | Is decentralization the future of sustainable energy?.pdfi, Available on: $\mathrm{https}$ ://ec.europa.eu/environment/integration/research/n ewsalert/pdf/145na4_en.pdf

9. G. Guérard, optimisation de la diffusion de l'énergie dans les smart grids, thèse de doctorat, (2014)

10. C. Petermann, S. Ben Amor et A. Bui, Approches théoriques pour la modélisation efficace de smart grid, Société française de recherche opérationnelle et d'aide à la décision, (2012)

11. H.K. Nguyen, J.B. Song, Z. Han, Demand side management to reduce peak-to-average ratio using game theory in smart grid, In 2012 Proceedings IEEE INFOCOM Workshops (pp. 91-96), IEEE, (2012, March)

12. H.T. Zhang, F.Y. Xu, L. Zhou, Artificial neural network for load forecasting in smart grid, In 2010 International Conference on Machine Learning and Cybernetics (Vol. 6, pp. 3200-3205), IEEE, (2010, July).

13. X. He, T. Huang, C. Li, H. Che, Z. Dong, A recurrent neural network for optimal real-time price in smart grid, Neurocomputing, 149, 608-612, (2015).

14. F. Mountassir, R. Mali et M. Bousmah, Machine learning au service de la prédiction de la demande d'énergie dans les smart grid, in Mediterranean Telecommunications Journal, vol.8, $\mathrm{n}^{\circ} 2$, (2018). 